\title{
Role of ionotropic glutamatergic receptors and nitric oxide in the effects of flutriafol, a triazole fungicide, on the in vivo striatal dopamine release
}

\author{
Lilian R. Ferreira Faro', Miguel Alfonso', Luis A. L. Maués ${ }^{1,2}$ and Rafael Durán \\ ${ }^{1}$ Department of Functional Biology and Helth Sciences, Faculty of Biology, University of Vigo, \\ Campus As Lagoas-Marcosende, Vigo 36310, Spain \\ 2Institute of Biological Sciences, Federal University of Pará, Rua Augusto Corrêa $S / N$, \\ Campus do Guamá, Belém-Pará 66075-900, Brazil
}

(Received May 28, 2012; Accepted September 5, 2012)

\begin{abstract}
Flutriafol is a triazole fungicide that induces spontaneous and depolarization-stimulated release of dopamine from rat striatum, although the neurochemical mechanism by which this fungicide induces this effect is unknown. The purpose of the present work was to assess the implication of ionotropic glutamatergic receptors and nitric oxide (NO) production in the flutriafol-induced dopamine release from rat striatum. To this, we have used non-competitive antagonists of NMDA (dizocilpine, MK-801), and (AMPA)/kainate (6-cyano-7-nitroquinoxaline-2,3-dione, CNQX) receptors, or nitric oxide synthase (NOS) inhibitors (Nomega-nitro-L-arginine -L-NARG - and 7-nitro-indazol - 7-NI), to study the striatal dopamine release induced by flutriafol. Intrastriatal infusion of $6 \mathrm{mM}$ flutriafol increased the dopamine levels to $984 \pm 141 \%$, with respect to basal levels. Infusion of flutriafol $(6 \mathrm{mM})$ in MK-801 $(500 \mu \mathrm{M})$ or CNQX $(500 \mu \mathrm{M})$ pretreated animals, increased striatal dopamine levels to $489 \pm 74 \%$ and $477 \pm 78 \%$, with respect to basal levels, respectively, these increases being $50.3 \%$ and $51.5 \%$ smaller than those induced by flutriafol in non-pretreated animals. Infusion of flutriafol $(6 \mathrm{mM})$ in L-NARG $(1 \mathrm{mM})$ or 7-NI $(100 \mu \mathrm{M})$ pretreated animals, increased the extracellular dopamine levels to $400 \pm 88.5$ and $479 \pm 69.4 \%$, with respect to basal levels, respectively, these increases being 59.3 and $51 \%$ smaller than those induced by flutriafol in non-pretreated animals. In summary, flutriafol appears to act, at least in part, through an overstimulation of NMDA receptors with possible NO production to induce dopamine release, and the administration of NMDA and AMPA/kainate receptor antagonists and NOS inhibitors protects against flutriafol-induced dopamine release from rat striatum.
\end{abstract}

Key words: Flutriafol, Triazole fungicides, Dopamine, Glutamatergic receptors, Nitric oxide synthase inhibitors, Brain microdialysis

\section{INTRODUCTION}

Flutriafol ([RS]-2,4'-difluoro- $\alpha$-[1H-1,2,4-triazol-1ylmethyl] benzhydryl alcohol) is a triazole plant protection fungicide which effectiveness in controlling several diseases affecting a wide range of crops has been proved. Triazole fungicides have a great importance in agriculture and medicine, being commonly used in different ways and in large quantities throughout the world. The intensive use of these compounds can generate a lot of residues that may potentially lead to substantial environmental contamination. So, triazole residues or triazole metabolites may occur in the environment and should be considered as a risk in terms of food, drinking water and non-occupational exposure.

However, in spite of their large use, comprehensive data assessing the neurotoxic effects of triazole fungicides in general, and flutriafol in particular, are almost lacking. The main part of the available data are published by regulatory agencies (e.g., Food and Agriculture OrganizationFAO, Environmental Protection Agency-EPA), and the toxicity evaluations have been performed only according to regulatory submission requirements. Some of these regulatory studies (summarized in the EPA report, 2006) pro-

Correspondence: Lilian R. Ferreira Faro (E-mail: lilianfaro@uvigo.es) 
pose that triazoles present various deleterious effects on mammalian biological systems, especially on the nervous system. This document by EPA (2006), reports evidences about the exposure to triazoles causing neurotoxicity, and neuropathological lesions in rat and mouse central and peripheral nervous system, as well as, a decrease in brain weight in both rats and mice.

On the other hand, systematic experimental studies have shown that a triazole fungicide, the triadimefon (and its metabolite the triadimenol), produces changes in dopaminergic neurotransmission. The effects of these substances on the dopaminergic system were described for the first time in late 1980s and early 1990s, with initial observations of neurotoxic effects of triadimefon on the motor activity (Crofton, 1996; Crofton et al., 1988, 1989; Moser and MacPhail, 1989; Walker et al., 1990). Several posterior studies, demonstrated that the behavioral effects produced by triadimefon were caused by changes in dopaminergic neurotransmission (Crofton, 1996; Hill et al., 2000; Ikaiddi et al., 1997; Reeves et al., 2003; Walker and Mailman, 1996). More recently, Gagnaire and Micillino (2006), examined the in vivo effects of administration of triadimefon in rats, and reported a significant increase in striatal dopamine extracellular levels induced by triazole. Gagnaire and Micillino (2006) argued that those increases in dopamine extracellular levels possibly occurred through an inhibition of dopamine transporter (DAT) induced by the triadimefon treatment.

A previous study from our laboratory has demonstrated that the in situ administration of flutriafol into rat striatum, also induces changes in dopaminergic neurotransmission (Faro et al., 2009; Santana et al., 2009). It was observed that intrastriatal administration of different concentrations of flutriafol produced significant concentration-dependent increases in dopamine extracellular levels measured by brain microdialysis (Santana et al., 2009). However, the authors suggest that, contrary to triadimefon, these increases in the in vivo dopamine release induced by flutriafol probably occurs due to the exocytotic release of neurotransmitter. So, despite the fact that triadimefon and flutriafol belong to the same chemical group, their mechanisms of action appear to be different.

Thus, flutriafol induces in vivo dopamine release from rat striatum, although the neurochemical mechanism by which fungicide induces exocytotic dopamine release from rat striatum is unknown. On the other hand, dopaminergic neurotransmission in the nigrostriatal pathway is regulated by some neurotransmitters including glutamate, acetylcholine and GABA. In the striatum, glutamate is released from glutamatergic neurons whose somas are in the cerebral cortex, constituting the cortico-striatal pathway. Several studies demonstrated the presence of glutamatergic receptors localized on dopaminergic terminals, and the activation of those receptors by glutamate or glutamate analogues can changes dopamine levels (David et al., 2005, for review).

For that reason, it was considered interesting to investigate the effects of the NMDA (N-methyl-D-aspartate) receptors antagonists $\mathrm{MK}-801$ (dizocilpine, a non-competitive antagonist), and AMPA ( $\alpha$-amino-3-hydroxy5-methyl-4-isoxazolepropionic acid)/kainate receptor antagonist CNQX (6-cyano-7-nitroquinoxaline-2,3-dione) on the flutriafol-induced in vivo dopamine, DOPAC (3,4-dihydroxyphenylacetic acid) and HVA (homovalic acid) release from rat striatum. In addition, to provide evidence for the proposed mechanism of flutriafol action, the inhibitors of nitric oxide synthase (NOS), L-NARG (Nomega-nitro-L-arginine) and 7-nitro-indazol (7-NI) were also examined.

\section{MATERIALS AND METHODS}

\section{Animals, drug treatments and experimental groups}

Female adult Sprague-Dawley rats $(240-260$ g, $\mathrm{n}=45)$ were used in all experiments. Animals were housed under controlled conditions of temperature $\left(22 \pm 2^{\circ} \mathrm{C}\right)$ and light (light:dark 14:10 hr), with free access to food and water. The experiments were performed according to the Guidelines of the European Union Council (86/609/EU) and the Spanish State (Real decreto 223/1988 and 1201/2005) for the use of laboratory animals. All possible efforts were made to avoid animal suffering and distress.

Flutriafol was dissolved in dimethylsulfoxide (DMSO) at a final DMSO concentration of $2.5 \%$ and applied locally in the striatum through the dialysis probe. All other drugs were dissolved in the perfusion fluid. Flutriafol was purchased from Pestanal ${ }^{\circledR}$ - Sigma-Aldrich (St. Louis, MO, USA); dopamine, DOPAC, HVA, MK-801, CNQX, L-NARG and 7-NI were purchased from Sigma (Seelze, Germany). All other chemicals and reagents were of analytical grade.

\section{Microdialysis procedure}

Brain microdialysis in freely moving rats was carried out as described previously (Santana et al., 2009). Rats were anaesthetized with chloral hydrate $(400 \mathrm{mg} / \mathrm{kg}$, i.p.), and guide cannulae (CMA/11, CMA/Microdialysis, Sweden) were implanted into the dorsal striatum, employing the stereotaxic coordinates (AP: $+1.0 \mathrm{~mm}$, LR: $+3.0 \mathrm{~mm}$, DV: $6 \mathrm{~mm}$ ) according to Paxinos and Watson 
(1998) stereotaxic atlas as a reference. Twenty four hours after surgery microdialysis probes $(3 \mathrm{~mm}$ long, $0.5 \mathrm{~mm}$ O.D. diameter PES membrane of 100-kDa cutoff; CMA/12, CMA/Microdialysis, Sweden) were inserted through the guide cannulae. The probes were perfused with a Ringer's solution $(147 \mathrm{mM} \mathrm{NaCl}, 4 \mathrm{mM} \mathrm{KCl}$, $3.4 \mathrm{mM} \mathrm{CaCl} ; \mathrm{pH} \mathrm{7.4)}$ at a rate of $1.5 \mu \mathrm{l} / \mathrm{min}$ using a CMA/102 infusion pump (CMA/Microdialysis, Sweden). Placement of probes within the striatum was confirmed histologically after the experiment, using the Paxinos and Watson (1998) stereotaxic atlas as a reference. Only data from animals with correct probe placements in the striatum were used.

All experiments were conducted using awake, conscious, and freely-moving animals. The experiments were carried out over periods of $4 \mathrm{hr}$, sampling striatal dialysates every $20 \mathrm{~min}(30 \mu \mathrm{l})$. After collecting three basal samples (60 min), flutriafol was infused for $60 \mathrm{~min}$; after this, the medium was switched back to the unmodified Ringer's solution and sampling was continued for an additional period of $120 \mathrm{~min}$. For the studies of the effects of antagonists or inhibitors, after collecting three basal samples, the different drugs were infused for $1 \mathrm{hr}$ and then mixed with flutriafol, which was infused throughout the third hour of the experiment. Next, the medium was switched back to the unmodified Ringer's solution and sampling continued for an additional period of $60 \mathrm{~min}$.

\section{HPLC-EC analysis}

The microdialyzed samples $(30 \mu \mathrm{l})$ were collected by a CMA/142 microsampler (CMA/Microdialysis, Sweden) and dopamine, DOPAC and HVA were quantified by HighPerformance Liquid Chromatography with electrochemical detection (HPLC-EC). The dialysates were injected $(20 \mu \mathrm{l})$ into a HPLC-EC system using a Rheodyne 7125 injection valve. The isocratic separation of dopamine and its metabolites was achieved using Spherisorb ODS-1 reversed phase columns (10 $\mu \mathrm{m}$ particle size) according to Durán et al. (1998). The eluent ( $\mathrm{pH} 3.5)$ was prepared as follows: $70 \mathrm{mM} \mathrm{KH_{2 }} \mathrm{PO}_{4}, 1 \mathrm{mM}$ octanesulfonic acid, $1 \mathrm{mM}$ EDTA, and 15\% methanol. Elution was carried out at a flow rate of $1.5 \mathrm{ml} / \mathrm{min}$ using a Jasco PU 1,580 pump. The dopamine, DOPAC and HVA detection was achieved using an ESA Coulochem III electrochemical detector (ESA, Chelmsford, MA, USA) at a potential of $+400 \mathrm{mV}$. All dates were analysed by Cromanec XP 1.0.4.

\section{Expression of results and statistics}

Data were corrected using the percentage of in vitro recovery for every microdialysis probe, which was similar for the different probes used: dopamine $=10.5 \pm$
$0.5 \% ;$ DOPAC $=12.1 \pm 0.4 \% ; \mathrm{HVA}=14.7 \pm 0.9 \%$. The average of concentrations of the substances in the two samples collected before the administration of treatment were considered as basal levels and were taken as $100 \%$ in order to compare the different responses of dopamine and its metabolites after every treatment. The results are shown as the mean \pm S.E.M. expressed as a percentage with respect to basal levels.

Statistical analysis of the results was performed by means of ANOVA and Student-Newman-Keuls multiple range test, considering the following significant differences: $* \mathrm{P}<0.05, * * \mathrm{P}<0.01$, and $* * * \mathrm{P}<0.001$ with respect to the basal levels; ${ }^{\mathrm{P}}<0.05$, ${ }^{\mathrm{b}} \mathrm{P}<0.01$, and $\mathrm{c} \mathrm{P}<0.001$ with respect to the $6 \mathrm{mM}$ flutriafol group.

\section{RESULTS}

\section{Behavioural signs}

Intrastriatal administration of flutriafol or drugs at the concentrations tested did not produce seizures, tremors, weight loss or other types of apparent behavioural or physiological dysfunctions.

\section{Effects of flutriafol on basal dopamine, DOPAC and HVA release}

Periodical control experiments have been made under our microdialysis conditions to confirm the basal values and the adequacy of our conditions. Basal levels of dopamine and its metabolites in dialysed samples were stable in control animals (non-treated rats). The mean of dopamine, DOPAC and HVA concentrations in the two samples collected before flutriafol or drugs administration was considered as the basal levels: $0.46 \pm 0.03,15.8 \pm 6$ and $17.8 \pm 4 \mathrm{ng} / 20 \mathrm{~min}$, respectively.

Intrastriatal infusion of $6 \mathrm{mM}$ flutriafol significantly increased the extracellular levels of dopamine to $984 \pm$ $141 \%$ and DOPAC to $172.4 \pm 13 \%$, with respect to basal values, and did not produce any significantly changes in the extracellular HVA levels. The data for the effects of flutriafol on striatal dopamine, DOPAC and HVA levels are plotted in Figs. 1-5 in order to compare them with the data obtained under other experimental conditions (treatments with antagonists or inhibitors together with flutriafol).

\section{Effect of NMDA receptor antagonists on flutriafol-induced dopamine release}

To examine the role of NMDA receptors in the flutriafol-induced increase in dopamine overflow, we perfused the specific NMDA receptors antagonist MK-801 through the dialysis probe alone and mixed with $6 \mathrm{mM}$ flutria- 
fol. So, the one-hour infusion of 200 or $500 \mu \mathrm{M}$ MK-801 had no significant effects on striatal dopamine levels (Fig. 1). Under $200 \mu \mathrm{M}$ MK-801 pretreatment, the infusion of $6 \mathrm{mM}$ flutriafol increased striatal dopamine levels to $895 \pm 183 \%$, with respect to basal, which was not significantly different from the one produced in animals not pretreated with MK-801 (data not shown). The infusion of $6 \mathrm{mM}$ flutriafol in $500 \mu \mathrm{M}$ MK-801 pretreated animals increased striatal dopamine levels to $489 \pm 74 \%$, with respect to basal levels (Fig. 1), being this increase $50 \%$ smaller than the one observed with $6 \mathrm{mM}$ flutriafol in non-pretreated animals.

\section{Effect of AMPA/kainate receptor antagonists on flutriafol-induced dopamine release}

In order to investigate the possible role of AMPA/kainate receptors in the flutriafol-induced dopamine release, the effect of this pesticide was studied in the presence of antagonist CNQX. One hour infusion of $500 \mu \mathrm{M}$ CNQX had no significant effects on striatal dopamine levels (Fig. 2). Under $500 \mu \mathrm{M}$ CNQX pretreatment, infusion of $6 \mathrm{mM}$ flutriafol increased striatal dopamine levels to $477 \pm 78 \%$, with respect to basal (Fig. 2), being this increase $51.5 \%$ smaller than that observed with $6 \mathrm{mM}$ flutriafol in non-pretreated animals.

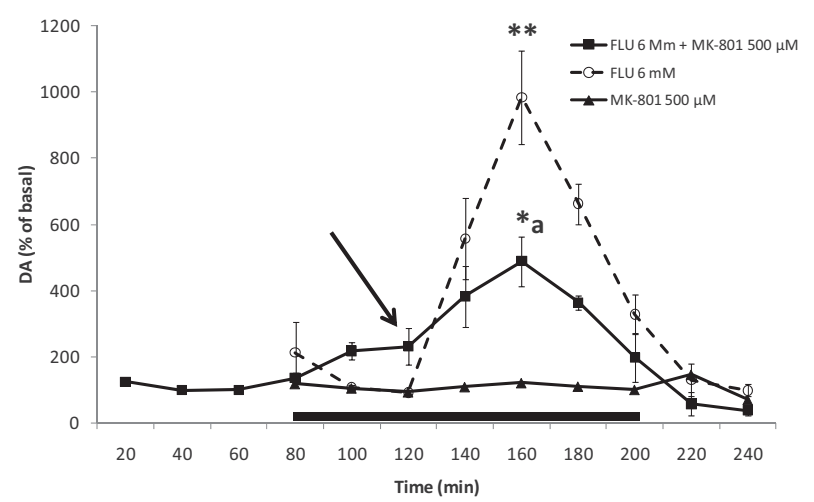

Fig. 1. Effect of $6 \mathrm{mM}$ flutriafol (FLU) infusion in MK-801 $(500 \mu \mathrm{M})$ pretreated rats on the dopamine (DA) extracellular levels from rat striatum. The MK-801 infusion is show by the black bar and flutriafol infusion started at the time indicated by the arrow over $60 \mathrm{~min}$. The results are shown as the mean \pm S.E.M., expressed as a percentage of basal levels (100\%). Basal levels were considered as the mean of dopamine concentrations in the two samples obtained before treatment administration. Significant differences were $* \mathrm{P}<0.05$ and $* * \mathrm{P}<0.01$, with respect to the basal and aP $<0.05$, with respect to the $6 \mathrm{mM}$ flutriafol control group.

\section{Effect of NOS inhibitors on flutriafol-induced dopamine release}

With the aim of investigating the implication of nitric oxide (NO) production in the effects produced by flutriafol on striatal dopamine levels, as well as the possible protective action of NOS inhibitors on flutriafol-induced dopamine release, we infused L-NARG or 7-NI through the microdialysis probe. Intrastriatal infusion of $1 \mathrm{mM}$ L-NARG for $1 \mathrm{hr}$ had no significant effects on striatal dopamine levels (Fig. 3). Under $1 \mathrm{mM} \mathrm{L-NARG} \mathrm{pre-}$ treatment, infusion of $6 \mathrm{mM}$ flutriafol increased striatal dopamine levels to $400 \pm 88.5 \%$, with respect to basal (Fig. 3), being this increase $51.3 \%$ smaller than the one observed with $6 \mathrm{mM}$ flutriafol in non-pretreated animals.

In the same way, $1 \mathrm{hr}$ intrastriatal infusion of $100 \mu \mathrm{M}$ 7-NI had no significant effects on striatal dopamine levels (Fig. 4). Under $100 \mu \mathrm{M}$ 7-NI pretreatment, the infusion of $6 \mathrm{mM}$ flutriafol increased striatal dopamine levels to $479 \pm 69.4 \%$, with respect to basal (Fig. 4), being this increase $\sim 60 \%$ smaller than that observed with $6 \mathrm{mM}$ flutriafol in non-pretreated animals.

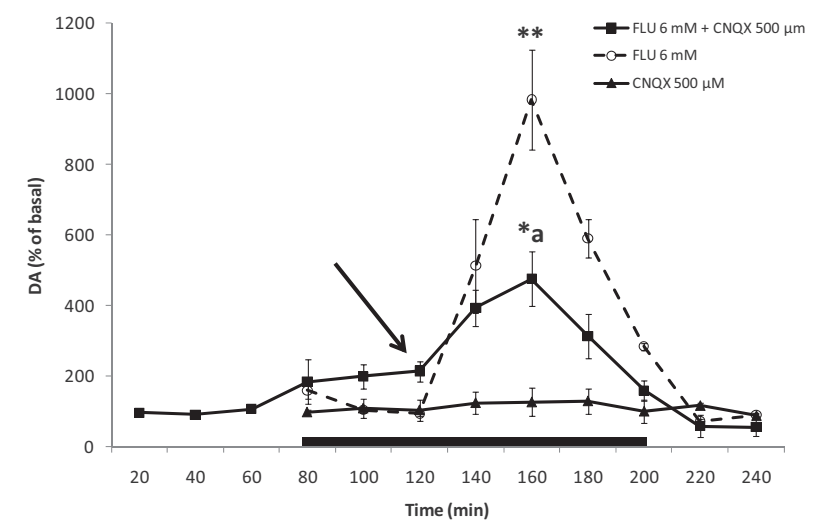

Fig. 2. Effects of $6 \mathrm{mM}$ flutriafol (FLU) infusion in CNQX $(500 \mu \mathrm{M})$ pretreated rats on the dopamine (DA) extracellular levels from rat striatum. The CNQX infusion is show by the black bar and flutriafol infusion started at the time indicated by the arrow over $60 \mathrm{~min}$. The results are shown as the mean \pm S.E.M., expressed as a percentage of basal levels (100\%). Basal levels were considered as the mean of dopamine concentrations in the two samples obtained before treatment administration. Significant differences were $* \mathrm{P}<0.05$ and $* * \mathrm{P}<0.01$, with respect to the basal and a $\mathrm{P}<0.05$, with respect to the $6 \mathrm{mM}$ flutriafol control group. 


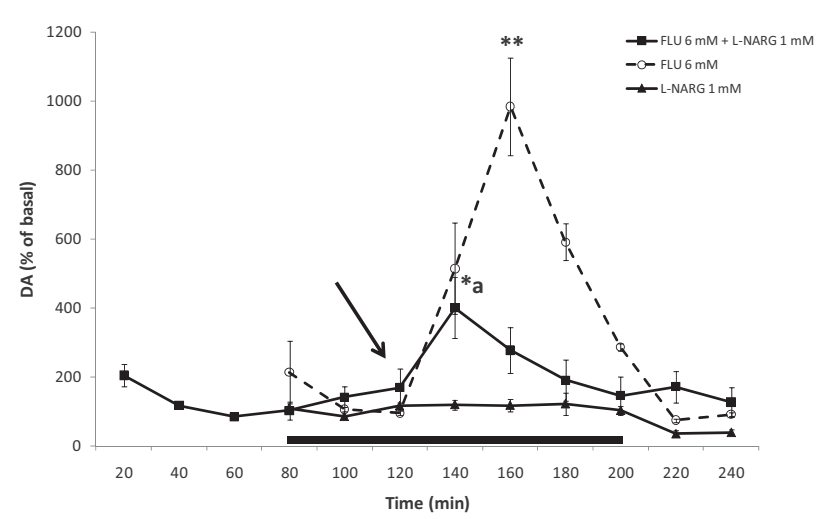

Fig. 3. Effects of $6 \mathrm{mM}$ flutriafol (FLU) infusion in L-NARG $(1 \mathrm{mM})$ pretreated rats on the dopamine (DA) extracellular levels from rat striatum. The L-NARG infusion is show by the black bar and flutriafol infusion started at the time indicated by the arrow over $60 \mathrm{~min}$. The results are shown as the mean \pm S.E.M., expressed as a percentage of basal levels (100\%). Basal levels were considered as the mean of dopamine concentrations in the two samples before treatment administration. Significant differences were $* \mathrm{P}<0.05$ and $* * \mathrm{P}<0.01$, with respect to the basal and $\mathrm{a}<<0.05$, with respect to the $6 \mathrm{mM}$ flutriafol control group.

\section{Effect of glutamatergic antagonists and NOS inhibitors on flutriafol-induced DOPAC and HVA release}

Fig. 5 illustrates the effects of $6 \mathrm{mM}$ flutriafol on extracellular levels of DOPAC and HVA, showing the maximal increases induced by pesticide and different treatments. Intrastriatal administration of MK-801 $(500 \mu \mathrm{M}), \mathrm{CNQX}$ $(500 \mu \mathrm{M})$, L-NARG $(1 \mathrm{mM})$, or 7-NI $(100 \mu \mathrm{M})$ during $1 \mathrm{hr}$ has non-significant effects on extracellular levels of DOPAC and HVA. Infusion of flutriafol in MK-801 or CNQX pretreated animals produced no change in the levels of both metabolites. In the same way, coinfusion of flutriafol and NOS inhibitors did not produce any change in the levels of DOPAC and HVA. Concentrations and time of perfusion of flutriafol and the different treatments were similar to the ones described in previous experiments with dopamine.

\section{DISCUSSION}

In previous striatal in vivo works, we observed that in situ administration of flutriafol increased both spontaneous and depolarization-evoked dopamine release from rat

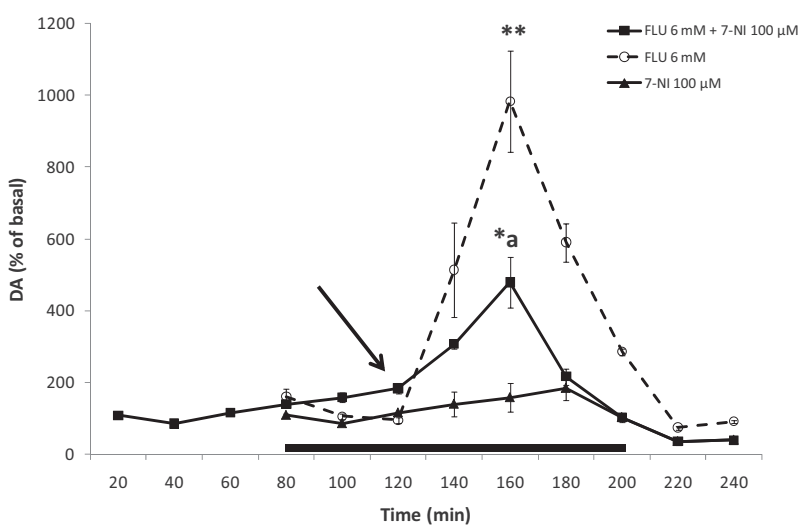

Fig. 4. Effects of $6 \mathrm{mM}$ flutriafol (FLU) infusion in 7-NI $(100 \mu \mathrm{M})$ pretreated rats on the dopamine (DA) extracellular levels from rat striatum. The 7-NI infusion is show by the black bar and flutriafol infusion started at the time indicated by the arrow over $60 \mathrm{~min}$. The results are shown as the mean \pm S.E.M., expressed as a percentage of basal levels (100\%). Basal levels were considered as the mean of dopamine concentrations in the two samples obtained before treatment administration. Significant differences were $* \mathrm{P}<0.05$ and $* * \mathrm{P}<0.01$, with respect to the basal and $\mathrm{aP}<0.05$, with respect to the $6 \mathrm{mM}$ flutriafol control group.

striatum (Faro et al., 2009; Santana et al., 2009). According to Santana et al. (2009), these effects probably occur due to an exocytotic release of dopamine, via a vesicular, $\mathrm{Ca}^{++}, \mathrm{Na}^{+}$and TTX-dependent mechanism. In the present work we have extended these in vivo studies to investigate the involvement of ionotropic glutamatergic receptors, NMDA and AMPA/kainate receptors, and NOS inhibitors, 7-NI and L-NARG, on the effects of flutriafol on striatal dopamine release.

In our experimental conditions, the one-hour administration of flutriafol $(6 \mathrm{mM})$ significantly increased striatal dopamine levels. The dopamine levels first increased and then recovered to the initial levels. This dopamine behaviour is consistent with the majority of microdialysis experiments, in which drugs are administered though a microdialysis probe. Furthermore, the concentration of flutriafol used in the study $(6 \mathrm{mM})$ was not high, since flutriafol was administered in situ through a dialysis membrane at a flow of $1.5 \mu \mathrm{l} / \mathrm{min}$ using a Ringer medium. Under these conditions, about $1.7 \%$ of flutriafol infused across the membrane reached the extracellular fluid of neuronal tissue (Santana et al., 2009). Thus, when we perfused $6 \mathrm{mM}$ flutriafol for a period of $60 \mathrm{~min}$, only $\sim 7.65 \mathrm{nmol}$ of flu- 

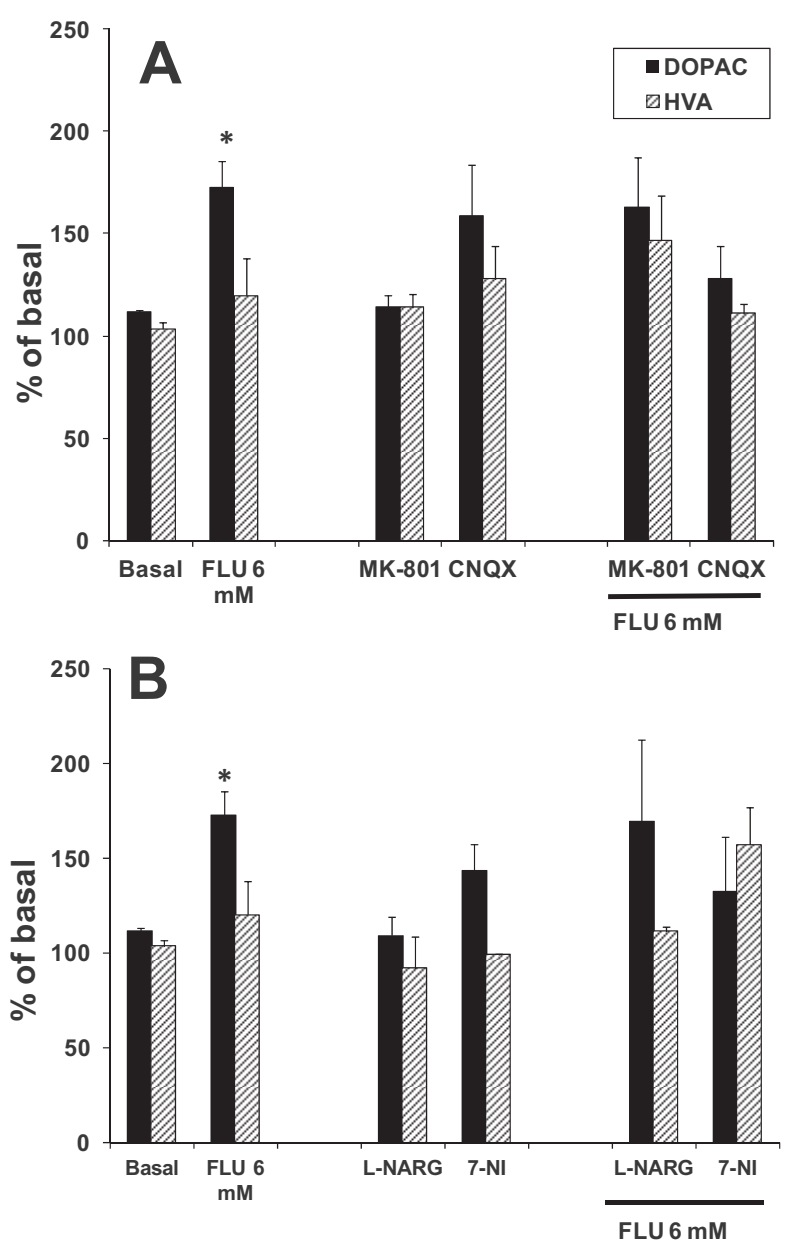

Fig. 5. Effects of intrastriatal perfusion of flutriafol $(6 \mathrm{mM})$ and different treatments on extracellular levels of DOPAC and HVA in rat striatum. Time of perfusion of flutriafol and the different treatments were similar to those in previous experiments with dopamine. The horizontal line indicates the perfusion of flutriafol in the different treatments. The results are shown as the mean \pm S.E.M. of 5-7 experiments, expressed as a percentage of basal levels (100\%). Basal levels were considered as the mean of DOPAC or HVA concentrations in the two samples obtained under treatment administration. Significant difference was $* \mathrm{P}<0.05$, with respect to the basal and ${ }^{\mathrm{P}}<0.05$, with respect to the $6 \mathrm{mM}$ flutriafol control group.

triafol were administered into the striatum.

The effect of flutriafol was studied in the presence of AMPA/kainate receptor antagonist (CNQX) and NMDA receptors antagonist (MK-801). So, pretreatment with $200 \mu \mathrm{M}$ MK-801 did not change the dopamine release induced by flutriafol, while intrastriatal administration of
$500 \mu \mathrm{M}$ MK-801 significantly decreased the effect of flutriafol over $50 \%$. Similar results were also observed with $500 \mu \mathrm{M}$ CNQX pretreatment that decreased the flutriafolinduced dopamine release over $51.5 \%$.

These results suggest that MK-801 and CNQX have the same inhibitory profile for flutriafol-induced dopamine release. However, our experimental conditions do not allow us to explain the reason for the similarity between the effects of both antagonists. To explain why both antagonists produced the same effect on flutriafolinduced dopamine-release would require a dose-response study of antagonists separately, to confirm whether or not the effect is similar. Further, in another set of experiments would require co-administer the two antagonists together with flutriafol into striatum to evaluate whether the effects are added. However, demonstrating the possible concentration-dependent effects of blockade of glutamate receptors was not the aim of this work, but to study the flutriafol-induced dopamine release.

As mentioned previously, the rat striatum is a brain area highly rich in NMDA and AMPA/kainate receptors that are present on striatal dopaminergic terminals (David et al., 2005). The enhancing of the dopamine release by the activation of these receptors was also evidenced. So, using in vivo microdialysis studies, it was observed that the increase of extracellular glutamate levels, or the perfusion of NMDA and AMPA evoked striatal dopamine release, being this effect inhibited by NMDA and AMPA/kainate receptor antagonists (Hernandez et al., 2003; Segovia et al., 1997; Mora et al., 2008 for review).

In line with these findings, we observed that both NMDA and AMPA/kainate receptors seemed to be involved in the in vivo dopamine release evoked by flutriafol in the rat striatum, because this release was attenuated by pretreatment of animals with NMDA or AMPA/ kainate receptors antagonists. Therefore, it seems likely that the flutriafol-induced increase in striatal dopamine overflow reflects an action of flutriafol on presynaptic ionotropic glutamate receptors located on nigrostriatal dopaminergic terminals. Another hypothesis would be that flutriafol could produce increases in extracellular glutamate levels though the stimulation of its release or an inhibition of its reuptake. This hypothesis is currently being studied in our laboratory, where the effects of flutriafol on the in vivo release of glutamate and other amino acid neurotransmitters in the striatum and cerebral cortex of rats are being evaluated.

It is also important to note that, although the concentration of the receptor antagonists used in these experiments should be sufficient to block the receptors (Campos et al., 2006; Faro et al., 2002; Vidal et al., 2007), we can- 
Role of iGluRs and NO in flutriafol-induced dopamine release

not ensure a complete blockade. However, the aim of this work was to demonstrate the participation of glutamatergic receptors on dopamine release, not to test the effects of total blockade of the same.

Because of the participation of NMDA receptors on dopamine release induced by flutriafol found in this study, and since NOS produces NO primarily in response to activation of NMDA receptors (Brenman and Bredt, 1997; Dawnson and Dawson, 1996; Garthwaite and Boulton, 1995; Schuman and Madison, 1994), in the next set of experiments we tested the role of NO production in the effect of flutriafol on in vivo dopamine release. We observed that pretreatment with L-NARG reduced flutriafol-induced dopamine release over $59.3 \%$, while the more specific neuronal NOS inhibitor, 7-NI, reduced release over $51 \%$. The sensitivity to both NOS inhibitors suggests that NO may facilitate the dopamine release induced by flutriafol.

The activation of NMDA receptors by glutamate increases $\mathrm{Ca}^{++}$entrance leading to a rise in their intracellular concentration. This $\mathrm{Ca}^{++}$binds to calmodulin and the $\mathrm{Ca}^{++}$/calmodulin complex activates NOS (Garthwaite et al., 1988) and NO production by nitrergic neurons (Shuman and Madison, 1994). On the other hand, it has been observed that the dopaminergic neurotransmission is influenced by NO in in vivo experiments (Segovia and Mora, 1998; West and Galloway, 1997a, 1997b). So, it has been shown that NO can stimulate or modulate the release of dopamine (Kiss, 2000; Kiss and Vizi, 2001; Kiss et al., 2004; Smith and Whitton, 2001).

The mechanism by which NO increases the striatal dopamine levels is not clear, although the same authors suggest that the mechanism through which the NO enhances dopamine release might be mediated by a reduction of dopamine transporter (DAT) activity, increasing the concentration of dopamine in the extracellular medium (Kiss et al., 1999, 2004; Lonart and Johnson, 1994; Pogun et al., 1994). The effect of NO on dopamine uptake allows NO to signal glutamatergic activity to the dopaminergic system through the change of inhibitory tone on transporters. This effect may increase the extracellular concentration of dopamine and this rise could represent the specific response of the dopaminergic system to the activation of glutamatergic neurotransmission. Thus, via NO, glutamate may participate in long-range nonsynaptic interactions, that is, NO can be regarded as a nonsynaptic extension of glutamate (Kiss and Vizi, 2001; Kiss et al., 2004).

According to the findings described here, we hypothesized that a stimulation of ionotropic glutamate receptors by flutriafol could be proposed as one possible neurotoxic mechanism underlying the effects of flutriafol on dopamine release. Moreover, the overstimulation of the NMDA glutamate receptors induced by flutriafol could produce an increase of intracellular $\mathrm{Ca}^{++}$concentration, and the activation of NOS and NO production. The NO diffuses to the extracellular medium and may reach the dopaminergic neurons and induce increases in levels of this neurotransmitter. So, the administration of NMDA antagonists or NOS inhibitors decreases the dopamine release in accordance with our results.

The fact that we only observed a partial ( $\sim 50-60 \%)$ reduction in flutriafol-induced dopamine release suggests that other mechanisms apart from NMDA or NOS activation may also be involved (for example, activation of AMPA/kainate receptors as observed by us in this study).

In terms of dopamine metabolites, flutriafol produced an increase in the extracellular levels of DOPAC and did not change the levels of HVA, with respect to basal levels. Considering that the primary extracellular DOPAC source comes from the metabolism, through MAO, of the newly synthesized cytoplasmic dopamine pool (Gazzara and Andersen, 1994; Zetterterstrom et al., 1988), a possible hypothesis that could explain this result would be that flutriafol, by the stimulation of the exocytotic release of dopamine, also stimulates the synthesis from tyrosine, increasing intracellular stocks of newly-synthesized dopamine. This way the increase of its metabolite levels would be explained. Pretreatment with MK-801, CNQX or NOS inhibitors did not change the DOPAC or HVA release induced by flutriafol. The lack of effect on metabolite levels could indicate that the metabolism of dopamine is not affected by these treatments.

In conclusion, flutriafol appears to act, at least in part, through an overstimulation of AMPA/kainate and NMDA receptors, possibly leading to an increase of intracellular $\mathrm{Ca}^{++}$and NO production to induce dopamine release. The administration of NMDA antagonist and NOS inhibitors partially blocked the flutriafol-induced dopamine release from rat striatum.

\section{ACKNOWLEDGMENTS}

Luiz Antonio Loureiro Maués acknowledges University of Vigo (Spain) for a research grant. The research was supported by grant (Contract-Program) from University of Vigo (0022 122F641.02).

\section{REFERENCES}

Brenmann, J.E. and Bredt, D.S. (1997): Synaptic signaling by nitric oxide. Curr. Opinion Neurobiol., 7, 374-378.

Campos, F., Alfonso, M., Vidal, L., Faro, L.R.F. and Durán, R. (2006): Mediation of glutamatergic receptors and nitric oxide 


\section{L.R.F. Faro et al.}

on striatal dopamine release evoked by anatoxin-a. An in vivo microdialysis study. Eur. J. Pharmacol., 548, 90-98.

Crofton, K.M. (1996): A structure-activity relationship for the neurotoxicity of triazole fungicides. Toxicol. Lett., 84, 155-159.

Crofton, K.M., Boncek, V.M. and Reiter, L.W. (1988): Hyperactivity induced by triadimefom, a triazole fungicide. Fundam. Appl. Toxicol., 10, 459-465.

Crofton, K.M., Boncek, V.M. and Macphail, R.C. (1989): Evidence for monoaminergic involvement in triadimefon-induced hyperactivity. Psychopharmacology, 97, 326-330.

David, H.N., Ansseau, M. and Abraini, J.H. (2005): Dopamineglutamate reciprocal modulation of release and motor responses in the rat caudate-putamen and nucleus accumbens of "intact" animals. Brain Res. Brain Res. Rev., 50, 336-360.

Dawson, V.L. and Dawson, T.M. (1996): Nitric oxide actions in neurochemistry. Neurochem. Int., 29, 97-110.

Durán, R., Alfonso, M. and Arias, B. (1998): Determination of biogenic amines in rat brain dialysates by high performance liquid chromatography. J. Liq. Chromotogr. Relat. Technol., 21, 27992811.

EPA-U.S. (2006): Environmental Protection Agency Pesticide. Reregistration eligibility decision for triadimefon and tolerance reassessment for triadimenol, Washington.

Faro, L.R.F., do Nascimento, J.L.M., Alfonso, M. and Durán, R. (2002): Protection of methylmercury effects on the in vivo dopamine release by NMDA receptor antagonists and nitric oxide synthase inhibitors. Neuropharmacology, 42, 612-618.

Faro, L.R.F., Alfonso, M., Cervantes, R. and Durán, R. (2009): Comparative effects of pesticides on in vivo dopamine release in freely moving rats. Basic Clin. Pharmacol. Toxicol., 105, 395-400.

Gagnaire, F. and Micillino, J.C. (2006): Effects of triadimefon on extracellular dopamine, DOPAC, HVA and 5-HIAA in adult rat striatum. Toxicology, 217, 91-104.

Gazzara, R.A. and Andersen, S.L. (1994): Calcium dependency and tetrodotoxin sensitivity of neostriatal dopamine release in 5 -day-old and adult rats as measured by in vivo microdialysis. J. Neurochem., 62, 1741-1749.

Garthwaite, J. and Boulton, C.L. (1995): Nitric oxide signaling in the central nervous system. Annu. Rev. Physiol., 57, 683-706.

Garthwaite, J., Charles, S.L. and Chess-Williams, R. (1988): Endothelium-derived relaxing factor release on activation of NMDA receptors suggests role as intercellular messenger in the brain. Nature, 336, 385-388.

Hernandez, L.F., Segovia, G. and Mora, F. (2003): Effects of activation of NMDA and AMPA glutamate receptors on the extracellular concentrations of dopamine, acetylcholine, and GABA in striatum of the awake rat: a microdialysis study. Neurochem. Res., 28, 1819-1827.

Hill, D., Ikaiddi, M., Mazzio, E. and Soliman, K.F. (2000): The neurochemical basis for the behavioral effects of triadimefon. Ann. N. Y. Acad. Sci., 914, 336-353.

Ikaiddi, M.U., Akunne, H.C. and Soliman, K.F. (1997): Behavioral and neurochemical effects of acute and repeated administration of triadimefon in the male rat. Neurotoxicology, 18, 771-780.

Kiss, J.P. (2000): Role of nitric oxide in the regulation of monoaminergic neurotransmission. Brain Res. Bull., 52, 459-466.

Kiss, J.P. and Vizi, E.S. (2001): Nitric oxide: a novel link between synaptic and nonsynaptic transmission. Trends Neurosci., 24, 211-215.

Kiss, J.P., Hennings, E.C., Zsilla, G. and Vizi, E.S. (1999): A possible role of nitric oxide in the regulation of dopamine transporter function in the striatum. Neurochem. Int., 34, 345-350.

Kiss, J.P., Zsilla, G. and Vizi, E.S. (2004): Inhibitory effect of nitric oxide on dopamine transporters: interneuronal communication without receptors. Neurochem. Int., 45, 485-489.

Lonart, G. and Johnson, K.M. (1994): Inhibitory effects of nitric oxide on the uptake of $3[3 \mathrm{H}]$ dopamine and $[3 \mathrm{H}]$ glutamate by striatal synaptosomes. J. Neurochem., 63, 2108-2117.

Mora, F., Segovia, G. and Del Arco, A. (2008): Glutamatedopamine-GABA interactions in the aging basal ganglia. Brain Res. Rev., 58, 340-353.

Moser, V.C. and MacPhail, R.C. (1989): Neurobehavioral effect of triadimefon, a triazole fungicide, in male and female rats. Neurotoxicol. Teratol., 11, 285-293.

Paxinos, G. and Watson, C. (1998): The Rat Brain in stereotaxic coordinates. Fourth Edition. Academic Press.

Pogun, S., Baumann, M.H. and Kuhar, M.J. (1994): Nitric oxide inhibits $[3 \mathrm{H}]$ dopamine uptake. Brain Res., 641, 83-91.

Reeves, R., Thiruchelvam, M., Richfield, E.K. and Cory-Slechta, D.A. (2003). Behavioral sensitization and long-term neurochemical alterations associated with the fungicide triadimefon. Pharmacol. Biochem. Behav., 76, 315-326.

Santana, M.B., Rodrigues, K.J.A., Durán, R., Alfonso, M., Vidal, L., Campos, F., De Oliveira, I.M. and Faro, L.R.F. (2009): Evaluation of the effects and mechanisms of action of flutriafol, a triazole fungicide, on striatal dopamine release by using in vivo microdialysis in freely moving rats. Ecotoxicol. Environ. Saf., 72, 1565-1571.

Schuman, E.M. and Madison, D.V. (1994): Nitric oxide and synaptic function. Annu. Rev. Neurosci., 17, 153-183.

Segovia, G. and Mora, F. (1998): Role of nitric oxide in modulating the release of dopamine, glutamate, and GABA in striatum of the freely moving rat. Brain Res. Bull., 45, 275-279.

Segovia, G., Del Arco, A. and Mora, F. (1997): Endogenous glutamate increases extracellular concentrations of dopamine, GABA, and taurine through NMDA and AMPA/kainate receptors in striatum of the freely moving rat: a microdialysis study. J. Neurochem., 69, 1476-1483.

Smith, J.C. and Whitton, P.S. (2001): The regulation of NMDAevoked dopamine release by nitric oxide in the frontal cortex and raphe nuclei of the freely moving rat. Brain Res., 889, 57-62.

Vidal, L., Durán, R., Faro L.R.F., Campos, F., Cervantes, R.C. and Alfonso, M. (2007): Protection from inorganic mercury effects on the in vivo dopamine release by ionotropic glutamate receptor antagonists and nitric oxide synthase inhibitors. Toxicology, 238, 140-146.

Walker, Q.D., Lewis, M.H., Crofton, K.M. and Mailman, R.B. (1990): Triadimefon, a triazole fungicide, induces stereotyped behavior and alters monoamine metabolism in rats Toxicol. Appl. Pharmacol., 102, 474-485.

Walker, Q.D. and Mailman, R.B. (1996): Triadimefon and triadimenol: Effects on Monoamine uptake and release. Toxicol. Appl. Pharmacol., 139, 227-233.

West, A.R. and Galloway, M.P. (1997a): Endogenous nitric oxide facilitates striatal dopamine and glutamate efflux in vivo: role of ionotropic glutamate receptor-dependent mechanisms. Neuropharmacol., 36, 1571-1581.

West, A.R. and Galloway, M.P. (1997b): Inhibition of glutamate reuptake potentiates endogenous nitric oxide-facilitated dopamine efflux in the rat striatum: an in vivo microdialysis study. Neurosci. Lett., 230, 21-24.

Zetterstrom, T., Sharp, T., Collin, A.K. and Ungerstedt, U. (1988): In vivo measurement of extracellular dopamine and DOPAC in rat striatum after various dopamina-releasing drugs; implications for the origin of extracelular DOPAC. Eur. J. Pharmacol., 148, 327-334. 